\title{
Transdisciplinary Higher Education-A Challenge for Public Health Science
}

\author{
Alexandra Krettek ${ }^{1,2}$ and Stefan Thorpenberg ${ }^{1,3}$ \\ ${ }^{1}$ Nordic School of Public Health, Box 12133, 40242 Gothenburg, Sweden \\ ${ }^{2}$ Department of Internal Medicine, Institute of Medicine, Sahlgrenska Academy at University of Gothenburg, \\ 40530 Gothenburg, Sweden \\ ${ }^{3}$ Research Policy Institute, Lund University, 22100 Lund, Sweden
}

Correspondence should be addressed to Alexandra Krettek, alexandra.krettek@nhv.se

Received 15 May 2011; Revised 15 June 2011; Accepted 21 June 2011

Academic Editor: Jan Elen

Copyright (C) 2011 A. Krettek and S. Thorpenberg. This is an open access article distributed under the Creative Commons Attribution License, which permits unrestricted use, distribution, and reproduction in any medium, provided the original work is properly cited.

\begin{abstract}
This paper highlights and discusses issues associated with transdisciplinary teaching and suggests ways to overcome the challenges posed by different epistemologies, methods, and ethical positions. Our own transdisciplinary teaching experience in public health helped us identify some important questions including (i) what is transdisciplinary research in practice, and does methods triangulation yield more valid results?, (ii) from a teaching perspective, how do biopsychosocial and medical research differ?, (iii) what is the difference between deductive and inductive research, and does each discipline represent a different ethical position?, and (iv) does pure inductive research lack theories, and does it require a hypothesis—a "rule of thumb" —on how to proceed? We also suggest ways to facilitate and enhance transdisciplinary teaching, focusing on what unites us and not on what sets us apart, openly underlining and highlighting our differences. Using diverse methodologies, a newly educated transdisciplinary workforce will likely extend current knowledge and facilitate solutions for complex public health issues.
\end{abstract}

\section{Introduction}

1.1. Challenges of New Research Policies That Focus on Transdisciplinary Action. During the last two decades, governments and funding agencies such as the European Commission have largely supported transdisciplinary research collaborations among several academic disciplines and nonacademic organisations. Theories like Mode 2 [1] and Triple Helix [2] support transdisciplinary policy. Compared to traditional research, Mode 2 communicates knowledge in the context of application. The Mode 2 method is transdisciplinary and hopefully is more socially accountable and reflexive. Triple Helix is rooted in democratic corporatism, wherein all social interests merge due to mutual interest in innovation and technological development. Both theories redefine the role of researchers as those who positively influence innovative and economic development and forsake their old role of neutrality and total objectivity.
Despite questions about whether it is possible to generalize these theories for all research and all circumstances, research policy has changed. Today's researchers are challenged to think in a transdicisciplinary mode, reaching beyond the borders of their own subject area to conduct "useful" research that benefits society. Still, current policies reflect a view of transdisciplinary research, not education; experience garnered from classical transdisciplinary disciplines does not play a substantial role in the debate. A limited focus on the transdisciplinary education, which is a prerequisite for training researchers in such thinking, has created a void.

Some view the transdisciplinary approach to research as abandoning the positivistic monodisciplinary academic culture. In the early 1950s, Rudolf Carnap was among the first to suggest combining skills and knowledge from different disciplines [3]. Even today, coexisting independent scientific cultures display a clear and continuing division 
between natural sciences and the humanities. Charles Percy Snow addressed this gap in his lecture, "The Two Cultures" [4]. Recent examples of transdisciplinary research include "Transdisciplinary Understanding and Training on ResearchPrimary Health Care," which aims to develop independent research scientists and increase the transdisciplinary focus in primary health care [5].

1.2. Public Health as Transdisciplinary Arena. Public health $(\mathrm{PH})$ has employed knowledge and methods from different areas (e.g., medicine and social sciences) since the 1880s. However, the epistemological differences between social sciences and medicine, and also between qualitative and quantitative methods, are quite large. While qualitative research often seeks new explanations based on preunderstanding, quantitative research frequently aims to verify its content by avoiding the "bias" of preunderstanding. However, most PH researchers were not trained in transdisciplinary thinking. Even as the debate on the differences between quantitative and qualitative methods becomes increasingly visible in the arena of $\mathrm{PH}$ education, the problems surrounding transdisciplinary education remain unresolved.

Increasingly complex global health problems necessitate continuous evolution of $\mathrm{PH}$ science as a transdisciplinary area. While health status has increased worldwide, its benefits are not shared equally. The widening gap between rich and poor nations exacerbates those inequities, pitting rural areas against cities, young people against old people, and men against women. Demographic changes in many lowand middle-income countries have created a double-disease burden of both communicable (infectious) and noncommunicable (lifestyle-related) diseases. Most often, health systems are ill prepared for complex scenarios, and funding to support ongoing health crises in politically unstable countries is often limited.

Thus, the earlier "one medicine" approach has given way to a global "one health" approach that provides $\mathrm{PH}$ with data about individuals, populations, and ecosystems data [6].

As new global health issues arise, newly recruited collaborators must be comfortable with cross-disciplinary interactions and understand the language, values, and behaviours of other disciplines. Unfortunately, the practical problems encountered in large transdisciplinary collaborations and their ensuing transdisciplinary teaching scenarios have received little attention in this context. While earlier publications in the field of transdisciplinary education were more abstract and theoretical [7], Godemann's recommendations for transdisciplinary cooperation are based on social psychology regarding information, knowledge transfer, and group dynamics [8]. A particularly vital issue involves engineering knowledge integration within heterogeneous, transdisciplinary groups. The success of this approach relies on information exchange, increased understanding, a common knowledge base, and awareness of the frame of reference. An important complimentary effect of transdisciplinary cooperation involves a group's increased flexibility in tackling group processes [8].
1.3. Transdisciplinary Teaching Is an Old Recipe That Steadily Receives New Interest. In his study on cholera at the Broad Street pump [9], John Snow employed both medical and social methods. Since then, PH science has recognized the need for different transdisciplinary and interdisciplinary approaches. We recently addressed this need in relation to obesity [10].

Some attempts to prepare students for cross-discipline collaborations (e.g., a recent evaluation of a transdisciplinary curriculum in health promotion) have been successful [11]. In graduate-level health education, transdisciplinary assignments provide greater understanding of the collaborating disciplines and enhance communication skills [12]. Using three conceptual approaches (i.e., adult learning theory, competency-based education, and an expanded Dreyfus model), a recent report suggested that $\mathrm{PH}$ education needs to orient its students to an outcome-based philosophy as it prepares them for the workforce [13]. Outcome-based education, which defines workforce expectations (learning outcomes/competencies) and then finds ways to achieve those expectations (knowledge, skills, and attitudes), is not new [14-16]. In general, education now emphasizes the synergy between teaching and learning outcomes.

A recent report focused on transdisciplinary education for health information professionals [17], illustrating yet again how higher education is trying to change in response to new requirements by academia and the labor market. Will it succeed?

The present paper highlights issues we encountered while teaching $\mathrm{PH}$ students from different disciplines. Four issues deserve particular attention:

(i) what is transdisciplinary research in practice, and does methods triangulation yield more valid results?

(ii) from a teaching perspective, how do biopsychosocial and medical research differ?

(iii) what is the difference between deductive and inductive research, and does each discipline represent a different ethical position?

(iv) does pure inductive research lack theories, or does it require a hypothesis—a "rule of thumb" —on how to proceed?

We also provide suggestions about facilitating and enhancing transdisciplinary teaching for students and teachers.

\section{Issues in Teaching Public Health Science}
2.1. What Is Transdisciplinary Research in Practice, and Does Methods Triangulation Yield More Valid Results? Due to its complexity and its social and economic impact on health, it is often said that PH science prefers a "triangulation" of methods [18]. Triangulation is understood as a blend of methods and theories from different disciplines. However, diverse methodology is often interpreted as a means for verifying findings and conclusions, an input of qualitative methods that will increase the truthfulness of a quantitative study. This interpretation may pose a problem. 
Our recent study on the swine flu outbreak in Västra Götaland, Sweden, provides insight on this issue. Because official records showed that the number of hospitalized patients did not exceed normal parameters, quantitative data supported a conclusion that no swine flu pandemic had occurred. However, interviews with hospital personnel revealed a stressful and out-of-ordinary work situation. Opposite quantitative and qualitative results demonstrated the real complexity: the pandemic was small in quantitative and biological terms, but extensive from a qualitative and social perspective [19]. Thus, a blend of methods can give different results but not falsify the first conclusion. Rather, the blend approach yielded a more complex understanding of the findings.

Therefore, the use of alternate methods may not improve the truth content of research results. If a mixture of methods is the criteria for creating true results, a blend would probably enhance most research results, even when they are incorrect. Since blended methods often yield new and deeper understanding, our experience in Västra Götaland will probably apply to other results in $\mathrm{PH}$ research.

The issue of triangulation has undergone extensive debate, especially regarding the practicality of combining qualitative and quantitative research methods for such purposes. Indeed, some researchers suggest that methodologies springing from different viewpoints are not interchangeable and therefore are not appropriate for blending in a triangulation of methods [20]. We believe that the methodologies complement each other well and should be used in concert due to the complexity of today's health issues, which require data and input from many perspectives [10].

Others assert that qualitative methods generate specific information that quantitative measures cannot provide, and that quantitative methods are better at finding small differences between groups. Regardless, the 1970s and 1980s debate that pitted qualitative against quantitative methodology debate is well behind us. Both methodologies have specific purposes and both extend knowledge rather than replace it or verify a theory in an absolute sense. The classroom will benefit from increased exposure to such thinking. For example, students could be asked to analyse study results using both qualitative and quantitative methodology. They will likely discover that the results differ slightly, so which result is truer? What different, but complimentary, angles are provided?

2.2. From a Teaching Perspective, How Do Biopsychosocial and Medical Research Differ? Another question for students involves whether health is something we have or something that happens. Depending on the specific discipline, health is characterized by the absence of illness or defined as something that encompasses anything outside and inside the physical body. Do these differing views create opportunities or hurdles? The former is a hallmark of the medical model, which views an individual as a case/patient. In the medical model, disease prevention uses surgery or drugs to normalize biomedical functions. Conversely, the biopsychosocial (health promotion) model views the individual as a whole person who exists within a social setting. The individual may or may not be ill, and many different factors (e.g., personality, beliefs, behaviors, and attitudes) affect her/his sense of illness or health. This difference in pedagogical approaches and concepts of health renders these two paradigms mutually exclusive [21].

Interestingly, much of today's health education curricula follow the medical model, focusing only on the presence or absence of disease and thus oversimplifying the concept of health. Nonetheless, the formation of interest groups like the Nordic Risk Group is encouraging because they postulate that the promotion of best practice requires health professionals to balance both humanistic and biomedical approaches to health, illness, and disease [22].

At the Nordic School of Public Health, one classroom strategy, designed to overcome the hurdle of varying definitions of health and illness, involves using teachers from different disciplines within the same course and encourages joint lectures and group discussions (e.g., senior lecturers from disease prevention, education, and health promotion jointly teaching a research course). Many thought provoking discussions have ensued when lecturers attend plenary discussions together, and each discipline presents its input on research methodologies, concepts, and the presence or absence of theory.

Another recent and innovative approach at the Nordic School of Public Health allowed two lecturers representing health promotion and cardiovascular disease (CVD) prevention, respectively, to deliver a joint lecture entitled "CVD in a PH Perspective," with lecturers alternating throughout the lecture. In our hands, these methods efficiently communicate a transdisciplinary approach by allowing students to observe the interaction between different disciplines in the classroom.

\subsection{What Is the Difference between Deductive and Inductive} Research, and Does Each Discipline Represent a Different Ethical Position? Disease prevention adheres to an empirical scientific approach (i.e., being inductive), while health promotion traditionally relies on a theoretical and conceptual approach (i.e., being deductive). Some students have difficulty understanding this distinction, and they often ask us for literature that discusses theories of health promotion, disease prevention, and treatment regimens.

Unfortunately, not all of these disciplines are based on theory. Disease prevention and treatment rely on empirical observation (i.e., "what works best"). In despair, one student questioned whether everything has to be based on theories and if we must always refer to ancient knowledge. Those comments are representative of students trained in health promotion or disease prevention. However, such beliefs present obstacles when students try to fit their results into existing theories, assuming that this must be done, and then find it difficult or even impossible. In our daily work, we repeatedly find that transdisciplinary teaching broadens the intellectual horizons of our students, forcing them to examine their results in a neutral and unbiased manner and to determine what they mean before trying to fit them into a box of previous knowledge. 
Moreover, subjects such as disease prevention, which originated in the traditional milieu of a medical faculty that follows the medical model, are often quantitative and expressed within the Anglo-Saxon scientific writing tradition. Conversely, health promotion, which is rooted in the social sciences, is often qualitative and frequently is communicated in the continental style of writing. This difference creates challenges when teachers apply different values and ways of expressing themselves while grading assignments, depending on their own writing tradition. Adding further complication, both writing traditions have different perceptions of science. The Anglo-Saxon model identifies science as an investigation based on problem solving, while the continental model perceives science as thinking and mostly theory based. Because early-career students have limited experience with scientific writing, mostly gained from the preferred style of one scientific discipline or even a nationally adopted "best way" of writing, they find such differences confusing. We also encounter these issues when we teach in different countries and cultures. Introducing both academic writing traditions to students can prevent either/or thinking.

A seldom-discussed but basic difference between quantitative and qualitative research involves research ethics. Ideally, a quantitative researcher often, but not always, starts without a preunderstanding of her/his subject, an approach that allows pure, inductive, and unbiased research. Conversely, the qualitative researcher accepts preunderstanding as an important part of the process but seldom uses the term "biased." Indeed, qualitative researchers sometimes use wording like "completely biased" to limit the impact of their preunderstanding [23]. Presenting examples that allow students to question the idea of an equal right to health against the norm of absolute objectivity and neutrality can be a powerful tool in the classroom. Such examples always initiate an interesting discussion, one that reveals the differing research ethics between quantitative and qualitative research.

\subsection{Does Pure Inductive Research Lack Theories, or Does It} Require a Hypothesis-a "Rule of Thumb"-on How to Proceed? In transdisciplinary teaching, labeling ideas as models, theories, and/or hypotheses in different disciplines creates confusion and fosters misunderstanding. Some students wonder if theories are limited to old ideas and if new studies merely result in new models, in the sense of works in progress. They ask, "When do we get new theories?".

In a Kuhnian paradigm [24], the existing "normal science" between revolutionary paradigm changes defines education in different research disciplines. As new theories appear, they are subject to questioning at an advanced level of higher education, which itself remains at the border of an imaginary research front.

Students vaguely understand what encompasses a theory. Sometimes they argue that practical $\mathrm{PH}$ research lacks theory, that it is only explorative and without hypotheses or preunderstanding. Students may need help in distinguishing between theories and hypotheses, where the latter are less defined but still have concrete meaning. On the other hand,
Kuhn defined paradigm in more diffuse terms that try to capture the inner content of research activities. According to heuristic principles [25], every research activity needs a general principal to guide the work. While different levels of preunderstanding may not require a known theory, they still need a defined hypothesis or a less visible paradigm-a general "rule of thumb" (i.e., a heuristic principle)—-to guide the research.

In humanist research and most social science, the generic position (i.e., research activities always have some preunderstanding) represents the typically biased view of many medical and natural science researchers. This view should be avoided. How, then, should this be taught in a transdisciplinary educational setting? What is right and what is wrong? Most likely, it can be both at the same time. We suggest that students sketch an anticipated research project (quantitative and/or qualitative) without using preunderstanding, heuristic principle, or even a hint of theoretical understanding. If they do that, they will see the problem.

\section{Discussion}

Researchers and teachers are constantly challenged to identify ways to enhance greater understanding between disciplines. What hurdles do we face? Are the differences we see superficial, masking a larger problem? Global acceptance of transdisciplinary approaches is critical. However, our own global experience in transdisciplinary teaching reveals a surprisingly uneven path. Although a few transdisciplinary educational experiments have been completed with positive effects on broadening knowledge [11, 12, 26, 27], the journey ahead is long.

To help develop future scientists who are familiar with and, most importantly, are able to use the methodological approaches and theoretical frames from different disciplines, we must communicate transdisciplinary thinking and methodologies early in the educational arena. Earlier reports identified several key components of transdisciplinary education [28] and discussed the challenges faced by research teams that include members from different disciplines [29].

Applying truly transdisciplinary teaching and research is possible only if participants engage in open dialogue and accept the notion that other scientific perspectives are equally important. Epistemological differences between quantitative and qualitative research aside, the most inductive researcher must start from some kind of preunderstanding of how to do the work. The qualitative researcher seeks new and unexpected results, even if he/she starts from a deductive question. The ideal of the neutral and objective researcher, often advocated by the epidemiology branch of $\mathrm{PH}$, is usually based on the understanding that research activities should provide society with long-term benefits. Conversely, the qualitative researcher, often found in health promotion, is probably not interested in supporting a governmental policy before determining its long-term impact on citizens.

Unfortunately, current health education is often limited to the medical model, which can oversimplify the assessment of a particular health problem. Current threats to $\mathrm{PH}$ demand that we stop limiting ourselves to one-dimensional 
teaching and problem solving. The future is now. We need to research, teach, and learn with broad and open minds now. Ideally, most of these activities are accomplished closer to the biopsychosocial model. Indeed, transdisciplinary teaching helps students develop a broader perspective and encourages them to consider their results in a neutral and unbiased way. Students must understand the challenges of interpreting data based on different disciplines and research methodologies and also master the process of preparing that data for publication in peer-reviewed journals. Everyone who teaches higher education courses in scientific writing and research methods should incorporate these challenges into their curricula.

Instilling our students with broad understanding is vitally important at all educational levels. Innovative educational tools that facilitate and enhance learning across disciplinary borders will help. Such tools require student awareness and a faculty that is ready to apply them (e.g., the joint lecturing already described). Another recent report describes a transdisciplinary teaching approach in nursing education [26].

Most importantly, all education should focus on outcome-based learning objectives. Indeed, all transdisciplinary efforts require understanding of the competencies students will need after they graduate and venture into the labor market. In 2000, the European Commission initiated a project entitled "TUNING Educational Structures in Europe," whose main goal involves universal learning outcomes/competencies for all higher education disciplines in Europe. In PH science, the Association of the Schools of Public Health is working to create joint competencies for a master of PH degree in the European region [30]. These competencies relate directly to the needs and requirements of the practical $\mathrm{PH}$ world. While this is a promising and important endeavor, hurdles remain. Given today's almost universal requirement for transdisciplinary research, academia should devote more attention to teaching in this context. Students need broad knowledge about a topic, but they also need help in developing their ability to think analytically and understand the scientific process inherent to both quantitative and qualitative methodologies. However, tailoring good transdisciplinary curricula (i.e., employing innovative pedagogy to efficiently transfer and integrate knowledge and learning opportunities across disciplines) requires an investment of time. Researchers also need time to enhance their understanding of the educational literature and learn about novel strategies for efficient delivery. Currently, research universities provide no incentive for properly valuing excellence in teaching, focusing instead on research [31].

Teaching could be viewed as a teacher/student partnership that shares, learns, and explores together [17] within a framework of relevant competencies. Teachers should train students in team skills relating to transdisciplinary environments. This could be accomplished using the recently described Learning in Teams model [32] and by applying the knowledge integration factors described by Goodemann [8].

While writing this paper, we found support for our ideas in "Education of Health Professionals for the 21st Century," the global commission that emphasises the necessity of reaching beyond national borders and individual disciplines to change the education of health professionals [33]. Therefore, our observations and suggestions are not only timely, but also give concrete examples of possible solutions in the area of $\mathrm{PH}$ science. Our examples are easily adaptable to other disciplines.

\section{Suggestions for Effective Transdisciplinary Teaching}

To facilitate and enhance transdisciplinary teaching for students and teachers, we suggest

(i) communicating the complexity of today's health issues and the concept of health in course curricula,

(ii) applying joint teaching efforts in plenary discussions and crafting lectures by teachers from different disciplines,

(iii) encouraging students to evaluate assignments and research projects using both quantitative and qualitative approaches,

(iv) enhancing interaction between participants from different disciplines through collaborative work in small groups,

(v) creating awareness of the differences between quantitative and qualitative research, especially regarding research ethics and academic writing traditions.

\section{Closing Thoughts}

We must foster good communication between different research cultures and academic disciplines. If we fail to bridge this gap, the goal of solving the world's $\mathrm{PH}$ problems is unattainable. All transdisciplinary teaching must focus on the things that unite us and set aside the things that set us apart, underlining and highlighting our differences rather than minimizing and hiding them. Today's new era requires us to educate students who will enter the new health workforce increasingly familiar with transdisciplinary thinking. The important question should always revolve around determining the common goal. Will the different methodologies help extend current knowledge and enable solutions for complex PH issues? Indeed, they will.

\section{Acknowledgments}

The authors thank their students for inspirational ideas and thought-provoking discussions, and Karen Williams for editorial expertise. This research received no specific grant from any funding agency in the public, commercial, or notfor-profit sectors.

\section{References}

[1] H. Nowotny, P Scott, and M. Gibbons, Re-Thinking Science: Knowledge And the Public in an Age of Uncertainty, Polity Press, Cambridge, UK, 2001. 
[2] H. Etzkowitz and L. Leydesdorff, Universities and the Global Knowledge Economy: A Triple Helix of University-IndustryGovernment Relations, Pinter, London, 1997.

[3] R. Carnap, "Logical foundations of the unity of science," in The Philosophy of Science, R. Boyd, P. Gasper, and J. Trout, Eds., The MIT Press, Cambridge, Mass, USA, 1991.

[4] C. P. Snow, Die Zwei Kulturen, Klett, Stuttgart, Germany, 1967.

[5] M. Stewart, G. Reid, J. B. Brown et al., "Development and implementation of training for interdisciplinary research in primary health care," Academic Medicine, vol. 85, no. 6, pp. 974-979, 2010.

[6] P. A. Conrad, J. A. Mazet, D. Clifford, C. Scott, and M. Wilkes, "Evolution of a transdisciplinary "One Medicine-One Health" approach to global health education at the University of California, Davis," Preventive Veterinary Medicine, vol. 92, no. 4, pp. 268-274, 2009.

[7] B. Nicolescu, "Towards transdisciplinary education," Transdisciplinary Research in Southern Africa, vol. 1, no. 1, pp. 5-15, 2005.

[8] J. Godemann, "Knowledge integration: a key challenge for transdisciplinary cooperation," Environmental Education Research, vol. 14, no. 6, pp. 625-641, 2008.

[9] J. Snow, On the Mode of Communication of Cholera, John Churchill, London, UK, 1855.

[10] J. Medin and A. Krettek, "An apple a day keeps the doctor away: interdisciplinary approaches to solving major public health threats," Scandinavian Journal of Public Health, vol. 36, no. 8, pp. 857-858, 2008.

[11] S. Misra, R. H. Harvey, D. Stokols et al., "Evaluating an interdisciplinary undergraduate training program in health promotion research," American Journal of Preventive Medicine, vol. 36, no. 4, pp. 358-365, 2009.

[12] C. Christie, A. R. Smith, and M. Bednarzyk, "Transdisciplinary assignments in graduate health education as a model for future collaboration," Journal of Allied Health, vol. 36, no. 2, pp. 6771, 2007.

[13] D. Koo and K. Miner, "Outcome-based workforce development and education in public health," Annual Review of Public Health, vol. 31, pp. 253-269, 2010.

[14] M. H. Davis, Z. Amin, J. P. Grande et al., "Case studies in outcome-based education," Medical Teacher, vol. 29, no. 7, pp. 717-722, 2007.

[15] R. M. Harden, "Outcome-based education: the future is today," Medical Teacher, vol. 29, no. 7, pp. 625-629, 2007.

[16] S. Mukhopadhyay and S. Smith, "Outcome-based education: principles and practice," Journal of Obstetrics and Gynaecology, vol. 30, no. 8, pp. 790-794, 2010.

[17] A. D. Cleveland, "Miles to go before we sleep: education, technology, and the changing paradigms in health information," Journal of the Medical Library Association, vol. 99, no. 1, pp. 61-69, 2011.

[18] N. Golafshani, "Understanding reliability and validity in qualitative research," The Qualitative Report, vol. 8, no. 4, pp. 597-607, 2003.

[19] L. Edgren, S. Thorpenberg, and B. Ahgren, "The new flu A (H1N1) in VGR - evaluation with an educational conceptual framework of the pandemic planning including the vaccination program in the Swedish county council of Region Västra Götaland," NHV Report 2010:10 R, Nordic School of Public Health, Göteborg, Sweden, 2010.

[20] J. E. M. Sale, L. H. Lohfeld, and K. Brazil, "Revisiting the quantitative-qualitative debate: implications for mixedmethods research," Quality and Quantity, vol. 36, no. 1, pp. 43-53, 2002.
[21] B. B. Jensen, "A case of two paradigms within health education," Health Education Research, vol. 12, no. 4, pp. 419-428, 1997.

[22] www.nordicriskgroup.com.

[23] R. J. MacCoun, "Biases in the interpretation and use of research results," Annual Review of Psychology, vol. 49, pp. 259287, 1998.

[24] T. S. Kuhn, The Structure of Scientific Revolutions, University of Chicago Press, Chicago, Ill, USA, 3rd edition, 1996.

[25] I. Lakatos, J. Worrall, and E. Zahar, Proofs and Refutations: The Logic of Mathematical Discovery, Cambridge University Press, Cambridge, UK, 1976.

[26] J. P. Griffin-Sobel, A. Acee, L. Sharoff, L. Cobus-Kuo, A. Woodstock-Wallace, and M. Dornbaum, "A transdisciplinary approach to faculty development in nursing education technology," Nursing Education Perspectives, vol. 31, no. 1, pp. 4143, 2010.

[27] D. Rapport, "Transdisciplinary education: where, when, how?" Ecosystems Health, vol. 4, no. 2, pp. 79-80, 1998.

[28] J. M. Nash, "Transdisciplinary training: key components and prerequisites for success," American Journal of Preventive Medicine, vol. 35, no. 2, supplement 1, pp. S133-S140, 2008.

[29] K. L. Hall, A. X. Feng, R. P. Moser, D. Stokols, and B. K. Taylor, "Moving the science of team science forward: collaboration and creativity," American Journal of Preventive Medicine, vol. 35, no. 2, supplement 1, pp. S243-S249, 2008.

[30] P. J. Whittaker, M. Pegorie, D. Read, C. A. Birt, and A. Foldspang, "Do academic competencies relate to 'real life' public health practice? A report from two exploratory workshops," European Journal of Public Health, vol. 20, no. 1, pp. $8-9,2010$.

[31] W. A. Anderson, U. Banerjee, C. L. Drennan et al., "Science education. Changing the culture of science education at research universities," Science, vol. 331, no. 6014, pp. 152-153, 2011.

[32] D. Magrane, O. Khan, Y. Pigeon, J. Leadley, and R. K. Grigsby, "Learning about teams by participating in teams," Academic Medicine, vol. 85, no. 8, pp. 1303-1311, 2010.

[33] J. Frenk, L. Chen, Z. A. Bhutta et al., "Health professionals for a new century: transforming education to strengthen health systems in an interdependent world," The Lancet, vol. 376, no. 9756, pp. 1923-1958, 2010. 


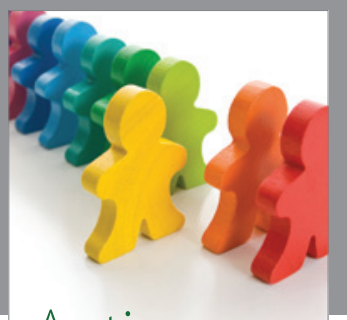

Autism

Research and Treatment
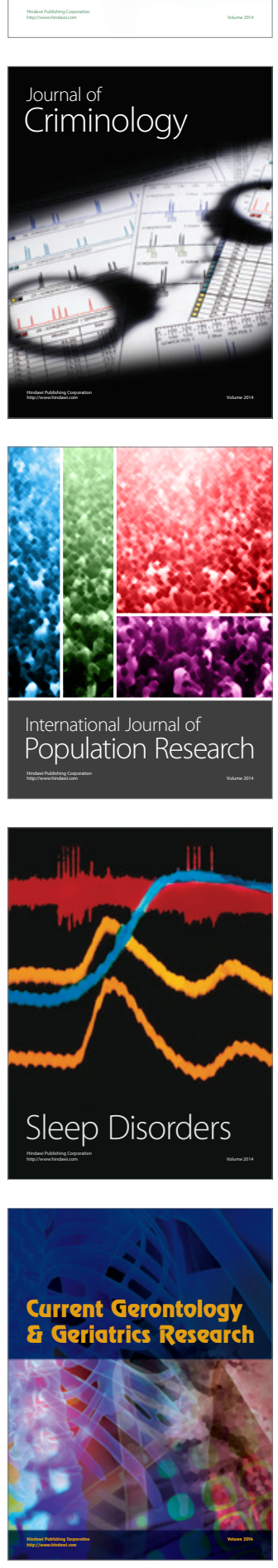
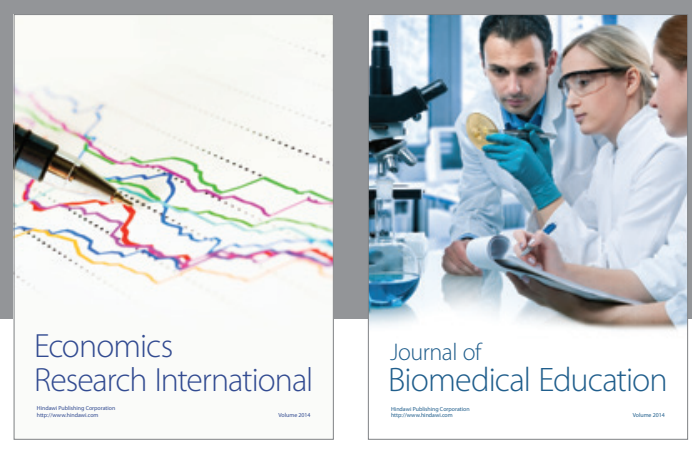

Journal of

Biomedical Education

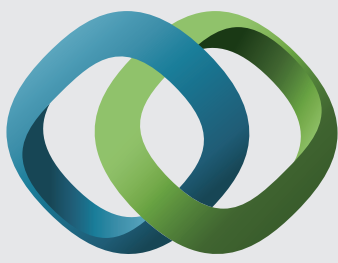

\section{Hindawi}

Submit your manuscripts at

http://www.hindawi.com
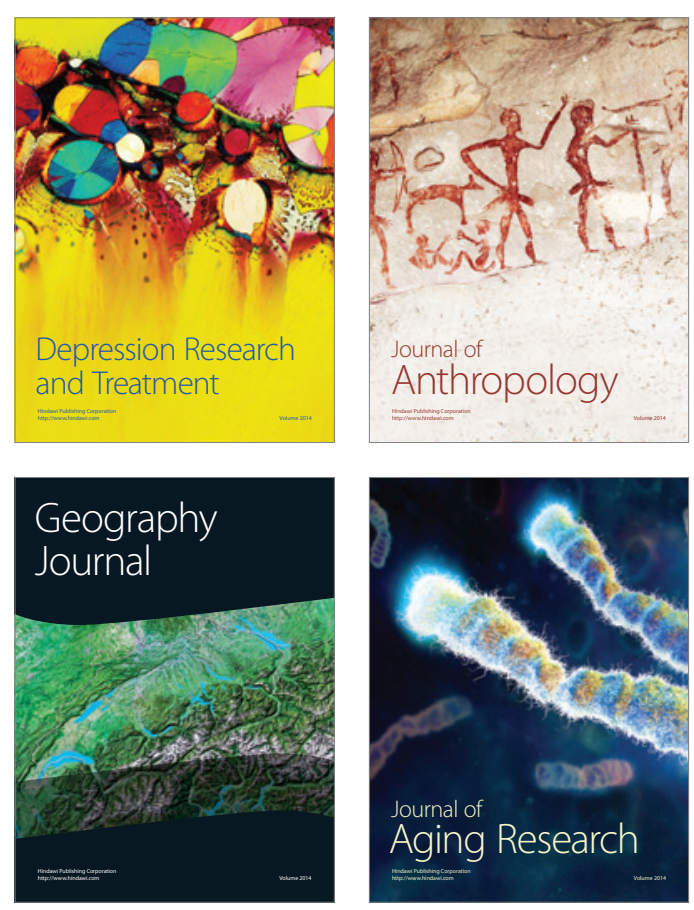

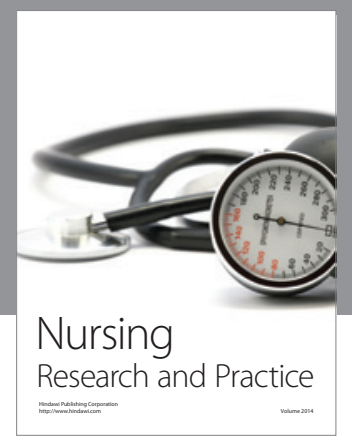

Nursing

Research and Practice

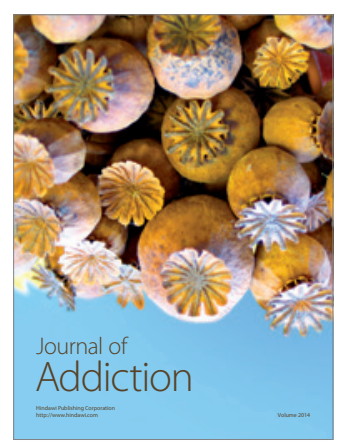

Child Development

Research

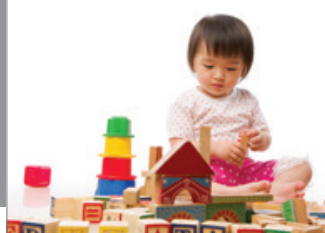

迥
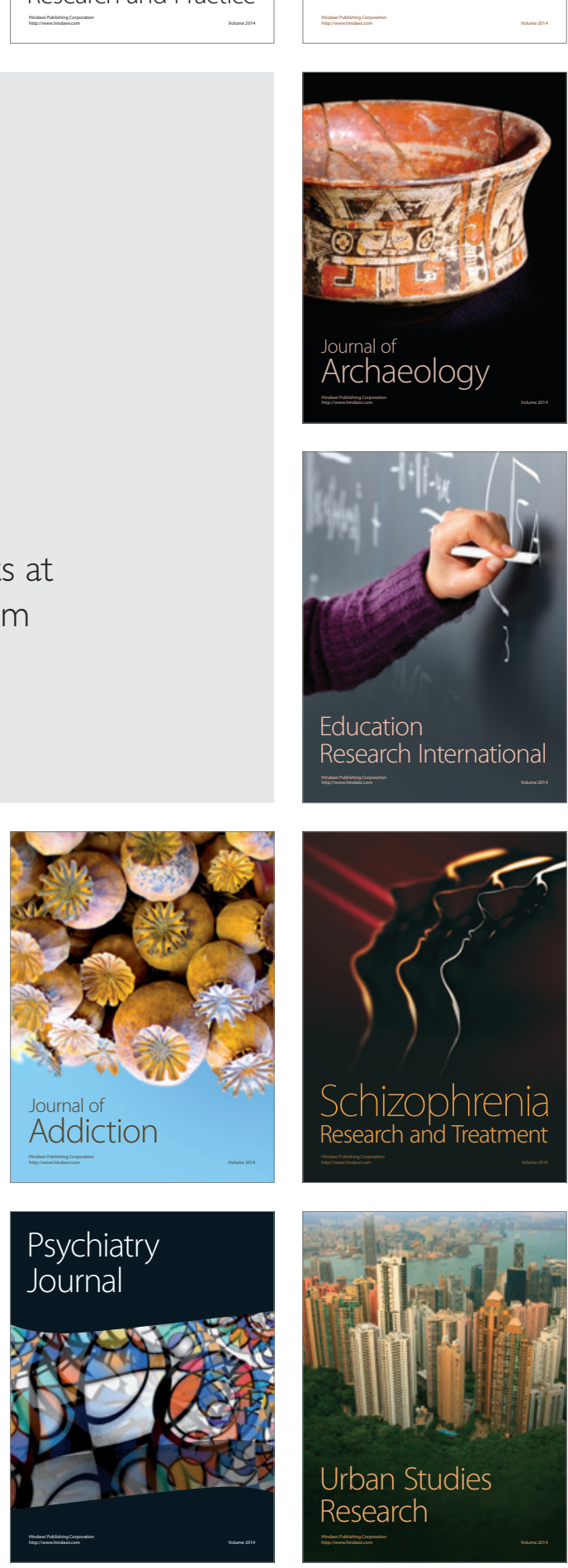